\title{
Is Type 2 Diabetes Causally Associated With Cancer Risk? Evidence From a Two-Sample Mendelian Randomization Study
}

\author{
Shuai Yuan,1,2 Siddhartha Kar, ${ }^{3}$ Paul Carter, ${ }^{4}$ Mathew Vithayathil,5 Amy M. Mason,6,7 Stephen Burgess, ${ }^{4,8}$ \\ and Susanna C. Larsson ${ }^{1,2}$
}

Diabetes 2020;69:1588-1596 | https://doi.org/10.2337/db20-0084

We conducted a two-sample Mendelian randomization study to investigate the causal associations of type 2 diabetes mellitus (T2DM) with risk of overall cancer and 22 site-specific cancers. Summary-level data for cancer were extracted from the Breast Cancer Association Consortium and UK Biobank. Genetic predisposition to T2DM was associated with higher odds of pancreatic, kidney, uterine, and cervical cancer and lower odds of esophageal cancer and melanoma but not associated with 16 other site-specific cancers or overall cancer. The odds ratios (ORs) were $1.13(95 \% \mathrm{Cl} 1.04,1.22), 1.08(1.00,1.17)$, 1.08 (1.01, 1.15), $1.07(1.01,1.15), 0.89(0.81,0.98)$, and 0.93 $(0.89,0.97)$ for pancreatic, kidney, uterine, cervical, and esophageal cancer and melanoma, respectively. The association between T2DM and pancreatic cancer was also observed in a meta-analysis of this and a previous Mendelian randomization study (OR 1.08; 95\% $\mathrm{Cl} 1.02,1.14 ; P=$ 0.009 ). There was limited evidence supporting causal associations between fasting glucose and cancer. Genetically predicted fasting insulin levels were positively associated with cancers of the uterus, kidney, pancreas, and lung. The current study found causal detrimental effects of T2DM on several cancers. We suggest reinforcing the cancer screening in T2DM patients to enable the early detection of cancer.

Type 2 diabetes mellitus (T2DM) and cancer are two major global health issues, causing approximately 5.0 and 8.7 million deaths and 143.0 and 208.3 million disability-adjusted life-years in 2015 worldwide, respectively $(1,2)$. Evidence from epidemiological studies indicates that T2DM is a risk factor for overall cancer (3) and several site-specific cancers, such as colorectal $(4,5)$, liver $(6)$, kidney $(7,8)$, uterine (9), and breast cancer (10). A bidirectional relationship has been suggested for T2DM and pancreatic cancer (11-15), whereas an inverse association has been observed between T2DM and risk of prostate cancer $(16,17)$. Findings for other site-specific cancers are conflicting (3), and the causality of the observed associations remains unclear due to possible residual confounding and reverse causality in observational studies.

Exploiting genetic variants as proxies for a risk factor, Mendelian randomization (MR) is a method that can strengthen the exposure-outcome association inference by diminishing the likelihood of confounding and eliminating reverse causality in conventional observational studies (18). This method minimizes confounding, since genetic variants are randomly assorted at conception, thereby being unrelated to self-adapted lifestyle and environmental factors. In addition, it overcomes reverse causality, as allelic randomization antedates the disease's onset.

Given the inconsistent results and potential methodological limitations of previous observational studies of T2DM and cancer risk, we conducted a two-sample MR study to assess the causal associations of liability to T2DM with
1Unit of Cardiovascular and Nutritional Epidemiology, Institute of Environmental Medicine, Karolinska Institutet, Stockholm, Sweden

2Department of Surgical Sciences, Uppsala University, Uppsala, Sweden

3MRC Integrative Epidemiology Unit, Bristol Medical School, University of Bristol, Bristol, U.K.

${ }^{4}$ Department of Public Health and Primary Care, University of Cambridge, Cambridge, U.K. 5MRC Cancer Unit, University of Cambridge, Cambridge, U.K.

${ }^{6}$ British Heart Foundation Cardiovascular Epidemiology Unit, Department of Public Health and Primary Care, University of Cambridge, Cambridge, U.K.

${ }^{7}$ National Institute for Health Research Cambridge Biomedical Research Centre, University of Cambridge and Cambridge University Hospitals, Cambridge, U.K. ${ }^{8}$ MRC Biostatistics Unit, University of Cambridge, Cambridge, U.K.
Corresponding author: Susanna C. Larsson, susanna.larsson@ki.se Received 22 January 2020 and accepted 25 April 2020

This article contains supplementary material online at https://doi.org/10.2337/ figshare.12200807.

(C) 2020 by the American Diabetes Association. Readers may use this article as long as the work is properly cited, the use is educational and not for profit, and the work is not altered. More information is available at https://www.diabetesjournals org/content/license. 
the risk of overall cancer and 22 site-specific cancers. For pancreatic cancer, a bidirectional MR study was conducted. We additionally explored the causal associations of genetically predicted fasting glucose (FG) and fasting insulin (FI) levels with the same cancer outcomes in secondary analyses. Moreover, we performed meta-analyses of available MR studies of the associations of T2DM, FG, or FI levels with cancer risk.

\section{RESEARCH DESIGN AND METHODS}

\section{Data Sources}

This two-sample MR study used summary-level genetic data from the DIAbetes Genetics Replication And Metaanalysis (DIAGRAM) consortium (19), Meta-Analyses of Glucose and Insulin-related traits Consortium (MAGIC) (20), Pancreatic Cancer Cohort Consortium (PanScan) and Pancreatic Cancer Case-Control Consortium (PanC4) (21), Breast Cancer Association Consortium (BCAC) (22), and UK Biobank (23) (Supplementary Table 1). Data for breast cancer came from BCAC and UK Biobank and were based on 228,951 European-descent participants (122,977 breast cancer case and 105,974 control subjects) and 367,643 European-descent participants (13,666 breast cancer case and 353,977 control subjects), respectively. The genomewide association study (GWAS) in BCAC used phase 3 of 1000 Genomes Project as a reference panel in the imputation stage and adjusted for genetic principal components and country. From UK Biobank, we additionally derived genetic association data, adjusted for age, sex, and 10 genetic principal components, for overall cancer and 21 other site-specific cancers among 367,643 unrelated participants. We identified a total of 75,037 cancer cases, and information on incident cancer cases was obtained until 31 March 2017 in UK Biobank. The cancer diagnosis source of included studies is shown in Supplementary Table 2. Most studies defined the cancer cases based on cancer registry or hospital/clinic data. The original GWAS had been approved by corresponding ethics committee, and the current study was approved by the Swedish Ethical Review Authority.

\section{Instrumental Variable Selection}

Instrumental variable selection for T2DM and FG and FI levels was based on a meta-analysis of 32 GWAS with 74,124 T2DM case and 824,006 control subjects of European ancestry (known as DIAGRAM consortium) (19) and a genome-wide association meta-analysis of up to 133,010 individuals of European ancestry without diabetes (known as MAGIC) (20), respectively. Instrumental variables for pancreatic cancer were obtained from a GWAS of 9,040 cancer case and 12,496 control subjects of European ancestry from PanScan and PanC4 (21). Single nucleotide polymorphisms (SNPs) that met the locus-wide significance level $\left(P<10^{-5}\right)$ and the genome-wide statistical significance threshold $\left(P<5 \times 10^{-8}\right)$ were proposed as instrumental variables for T2DM $(n=403), \mathrm{FG}(n=35)$, FI $(n=18)$, and pancreatic cancer $(n=22)$. Selected SNPs explained approximately $17.4 \%, 4.8 \%$, and $1.2 \%$ variance associated with T2DM, FG, and FI, respectively. Used instrumental variables for T2DM and glycemic traits were validated in previous studies (24-26). Previous studies reported that the effects of T2DM- and FI-related genetic variants in the FTO gene were entirely driven by BMImediation effects $(20,27)$. Thus, we excluded SNPs in or near the FTO gene region, leaving 399 SNPs as instrumental variables for T2DM, 35 for FG, and 17 for FI. With regard to T2DM, 295 SNPs (variants in FTO excluded) reaching the genome-wide significance level were used in the sensitivity analysis. Detailed information for instrumental variables of T2DM, FG, FI, and pancreatic cancer is presented in Supplementary Table 3 and Supplementary Table 4.

\section{Meta-analysis of MR Studies}

The procedure of systematic review and literature selection is shown in Supplementary Fig. 1. A systematic literature search was conducted in two data sets of PubMed and Embase. We identified 165 manuscripts published before 17 October 2019 by use of the following medical subject heading terms and/or text words: "diabetes," "glucose," "insulin," "glycemic," "cancer," "carcinoma," "Mendelian randomization," "Mendelian randomization," "instrumental variable causal inference," "causal inference using instrumental variable," and "causal inference using genetic variants." After title, abstract, and full text screening, seven studies were included in this meta-analysis (28-34). Details of exclusion criteria are presented in Supplementary Fig. 1. We extracted data of publication (the first author's name and year of publication), T2DM and related traits (FG and FI), cancer site, number of cancer case and control subjects, number of SNPs used as instrumental variables, variance explained by used SNPs, and risk estimates with their corresponding CIs. Information of included studies is shown in Supplementary Table 5.

\section{Statistical Analysis}

The random-effects inverse variance-weighted method was used to assess the associations of genetically predicted T2DM, FG, and FI with overall cancer and 22 site-specific cancers. Cochran I ${ }^{2}$ statistic was used to measure heterogeneity among instrumental variables. For T2DM, three sensitivity analyses, including the weighted median, MR-Egger, and MR Pleiotropy RESidual Sum and Outlier (MR-PRESSO) methods, were performed for the associations that showed suggestive evidence of associations in the inverse variance-weighted analysis. The weighted median approach provides accurate estimates with the prerequisite that at least half of the instrumental variables are valid (35). The MR-Egger regression detects and adjusts for pleiotropy; however, the derived estimates are imprecise (36). The MR-PRESSO method is able to detect and correct for possible outliers, thereby removing horizontal pleiotropy via outlier removal (37). To minimize the influence from BMI, we used a multivariable MR method 
with the adjustment of BMI. In the meta-analysis, effect sizes from different MR studies were combined using fixed-effects meta-analysis. Odds ratios (ORs) and CIs of cancer were scaled to 1-unit increase in log odds of liability to T2DM and 1-SD increase in log of genetically predicted FG and FI levels. The SD of FG and FI corresponds to $0.65 \mathrm{mmol} / \mathrm{L}$ and $0.60 \mathrm{pmol} / \mathrm{L}$, respectively, based on the Fenland or Ely study $(38,39)$. For pancreatic cancer, we additionally performed a bidirectional MR analysis. Power calculation for the analyses of T2DM was based on a web tool (40), and results are displayed in Supplementary Table 6. All statistical tests were two sided and performed in Stata/SE 15.0 and R 3.6.0 software. We did not use $P$ values strictly to define statistical significance but interpreted the results based on the strengths of the associations (41) as well as the consistency across sensitivity analyses.

\section{Data and Resource Availability}

Data for T2DM-associated SNPs can be obtained from the DIAGRAM consortium (https://diagram-consortium.org/ index.html). Data for FG- and FI-associated SNPs can be obtained from MAGIC (https://www.magicinvestigators .org/). Summary-level data from BCAC are publicly available (http://bcac.ccge.medschl.cam.ac.uk/). The PanScan and $\mathrm{PanC} 4$ genome-wide association data are available through dbGaP (accession numbers phs000206.v5.p3 and phs000648.v1.p1, respectively). UK Biobank data are available through application (https://www.ukbiobank.ac.uk/). Summary-level data for the used SNPs in the current study are available upon a reasonable request to the corresponding author.

\section{RESULTS}

We found no MR evidence of association between genetic liability to T2DM and overall cancer in the primary analysis or the sensitivity analyses (Fig. 1). However, there was some evidence of associations of genetic liability to T2DM with higher odds of liver, pancreatic, kidney, uterine, and cervical cancer and lower odds of melanoma and esophageal cancer (Fig. 2). The ORs per 1-unit increase in genetically predicted log odds of T2DM were 1.16 (95\% CI 0.99, 1.36; $P=0.059)$ for liver cancer, 1.13 (95\% CI 1.04, 1.22; $P=$ 0.002) for pancreatic cancer, 1.08 (95\% CI 1.00, 1.17; $P=0.039)$ for kidney cancer, 1.08 (95\% CI 1.01, 1.15; $P=0.031$ ) for uterine cancer, 1.07 (95\% CI 1.01, 1.15; $P=$ 0.031 ) for cervical cancer, 0.93 (95\% CI $0.89,0.97$; $P=0.001$ ) for melanoma, and 0.89 (95\% CI 0.81, 0.98; $P=0.018$ ) for esophageal cancer (Fig. 2). Estimates of similar magnitude were observed between genetic liability to T2DM and thyroid cancer (OR 1.08; 95\% CI 0.94, 1.24; $P=$ 0.281 ) and brain cancer (OR 0.92; 95\% CI 0.84, 1.02; $P=$ 0.104) (Fig. 2). The findings were consistent between analyses using 399 SNPs and 295 SNPs for T2DM (Supplementary Fig. 2). Results of sensitivity analyses showed same patterns in the analysis of esophageal and pancreatic cancer and melanoma (Fig. 3). We detected significant heterogeneity in the analysis of uterine and liver cancer and melanoma and pleiotropy in the MR-Egger analysis of cervical cancer. After outlier removal, all significant associations obtained from the inverse variance-weighted model remained in the MR-PRESSO analysis. In addition, a suggestive positive association between genetically predicted risk of pancreatic cancer and T2DM was observed in the reverse MR analysis (Supplementary Fig. 3). After adjustment for BMI, the patterns of the associations between genetically predicted log odds of T2DM and cancers remained albeit with wider CIs (Supplementary Fig. 4).

In the meta-analysis combining the present MR findings with those of previous MR studies (Supplementary Table 5), an association was observed between genetically predicted log odds of T2DM and pancreatic cancer (OR 1.08; 95\% CI 1.02, 1.14; $P=0.009$ ) among a total of 8,374 pancreatic cancer cases. The results of meta-analysis showed no associations of genetically predicted log odds of T2DM with kidney, uterine, or ovarian cancer (Fig. 4).

There was limited evidence of associations of genetically predicted FG and FI levels with overall cancer and the 22 site-specific cancers (Supplementary Fig. 5, Supplementary Fig. 6, and Supplementary Fig. 7). However, the

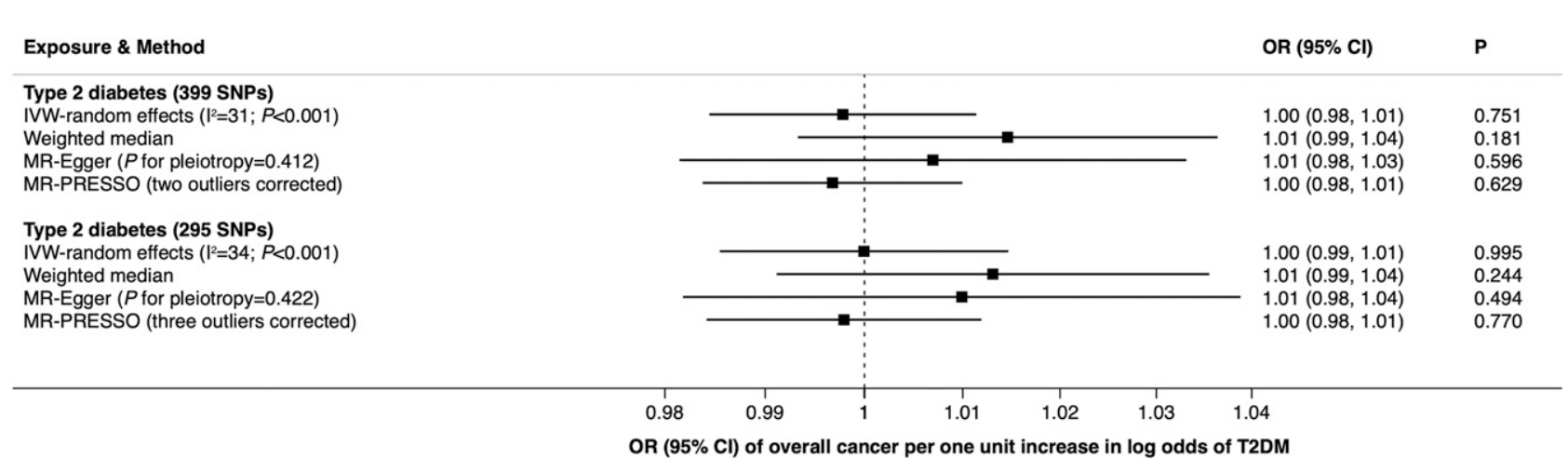

Figure 1-Association between T2DM and overall cancer in UK Biobank with 75,037 cancer patients and 292,606 participants without cancer. Heterogeneity was observed in both analyses. There was no detected pleiotropy in MR-Egger analyses. Two and three outliers were detected and corrected in the MR-PRESSO analysis using 399 SNPs and 295 SNPs for T2DM, respectively. IVW, inverse variance weighted. 


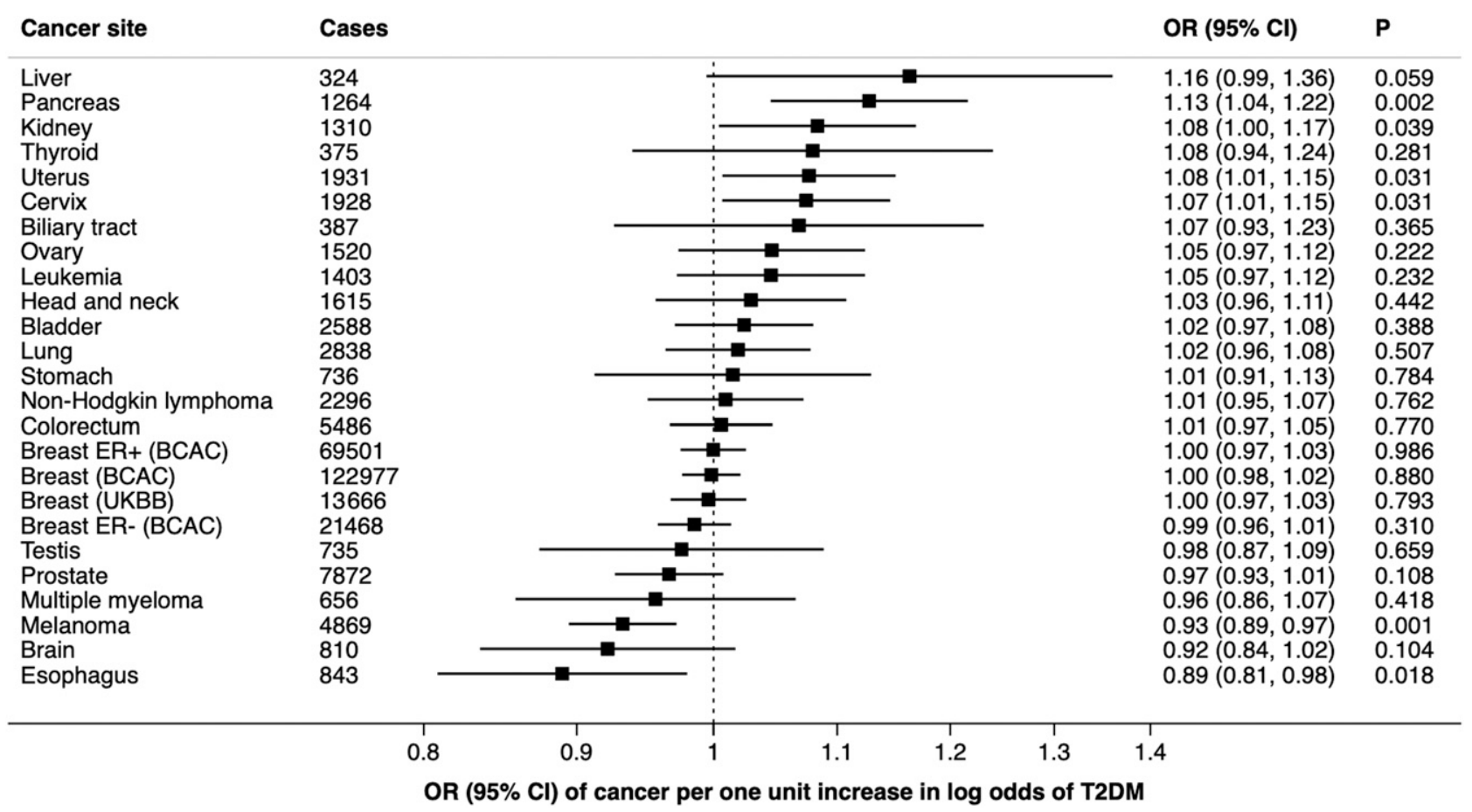

Figure 2-Associations between T2DM (399 SNPs) and 22 site-specific cancers in UK Biobank. All estimations were based on the inverse variance weighted method. ER, estrogen receptor; UKBB, UK Biobank.

precision was low in most analyses and the magnitude of the estimates was relatively strong for some cancer sites. For example, the OR was $>1.5$ for genetically predicted high FG levels in relation to biliary tract cancer (Supplementary Fig. 6). In addition, for FI levels, the ORs were $>1.5$ for kidney, uterine, cervical, and stomach cancer and $<0.5$ for liver cancer (Supplementary Fig. 7).

In the meta-analysis, there was no evidence of association between genetically predicted FG levels and five sitespecific cancers (Supplementary Fig. 8). Genetically predicted FI levels showed evidence of positive associations with cancers of the pancreas, kidney, uterus, and lung with combination of the findings from this MR study and previous MR studies (Supplementary Fig. 9).

\section{DISCUSSION}

The current study is the first MR study that has systematically investigated the causal associations of genetic liability to T2DM and related traits with overall cancer and 22 site-specific cancers. We found evidence that genetic liability to T2DM was associated with increased risks of pancreatic, kidney, uterine, and cervical cancer and with lower risks of melanoma and esophageal cancer. The positive association between genetic liability to T2DM and pancreatic cancer was further verified in a supplementary meta-analysis of MR studies. There was limited MR evidence supporting causal associations between genetically predicted FG and any cancer but genetically predicted high FI levels increased the risks of pancreatic, kidney, uterine, and lung cancer.
The present MR findings do not support observational studies suggesting an elevated risk of overall cancer among T2DM patients (3). An umbrella meta-analysis of 27 studies found that having T2DM was associated with a $10 \%$ higher risk of developing cancer (38,010 cancer cases) and a $16 \%$ higher cancer mortality rate (11,386 cancer-caused deaths) (3). In a national register-based cohort study in Australia, the standardized incidence and mortality ratios for all cancers combined were significantly higher (ORs ranging from 1.03 to 1.22 ) among both men and women with T2DM than in individuals without diabetes (16). However, our findings were in line with a recent individual-level MR study with 10,536 Japanese adults (3,541 cancer cases). Using 29 SNPs as instrumental variables for T2DM, that study found no strong evidence supporting an association between T2DM and overall cancer (42). The discrepancy with our overall cancer findings may be explained by the driver effects of the T2DM-unrelated cancers that contributed a large proportion of cancer cases, including breast cancer (18\%), prostate cancer (10\%), and colorectal cancer $(7 \%)$, in the present MR study, or from residual confounding or reverse causation bias in the observational studies.

Findings of the present MR study and the meta-analysis of MR studies showed a consistent causal positive association between T2DM and pancreatic cancer, supporting observational studies. An umbrella meta-analysis of 27 studies obtained a pooled OR of 1.95 when comparing T2DM patients with control subjects based on 52,445 pancreatic cancer cases (3). It has been demonstrated that 
OR $(95 \% \mathrm{Cl}) \quad P$

Uterus

IVW-random effects $\left(I^{2}=15 ; P=0.009\right)$

Weighted median

MR-Egger ( $P$ for pleiotropy $=0.144)$

MR-PRESSO (no outliers detected)

Cervix

IVW-random effects $\left(I^{2}=9 ; P=0.078\right)$

Weighted median

MR-Egger ( $P$ for pleiotropy $=0.002$ )

MR-PRESSO (one outlier corrected)

Kidney

IVW-random effects $\left(I^{2}=4 ; P=0.297\right)$

Weighted median

MR-Egger ( $P$ for pleiotropy $=0.269$ )

MR-PRESSO (no outliers detected)

Esophagus

IVW-random effects $\left(I^{2}=7 ; P=0.149\right)$

Weighted median

MR-Egger ( $P$ for pleiotropy $=0.277$ )

MR-PRESSO (no outliers detected)

\section{Pancreas}

IVW-random effects $\left(I^{2}=0 ; P=0.487\right)$

Weighted median

MR-Egger ( $P$ for pleiotropy $=0.173$ )

MR-PRESSO (no outliers detected)

Liver

IVW-random effects $\left(I^{2}=11 ; P=0.044\right)$

Weighted median

MR-Egger ( $P$ for pleiotropy $=0.865$ )

MR-PRESSO (three outliers corrected)

Melanoma

IVW-random effects $\left(I^{2}=12 ; P=0.031\right)$

Weighted median

MR-Egger ( $P$ for pleiotropy $=0.630$ )

MR-PRESSO (no outliers detected)

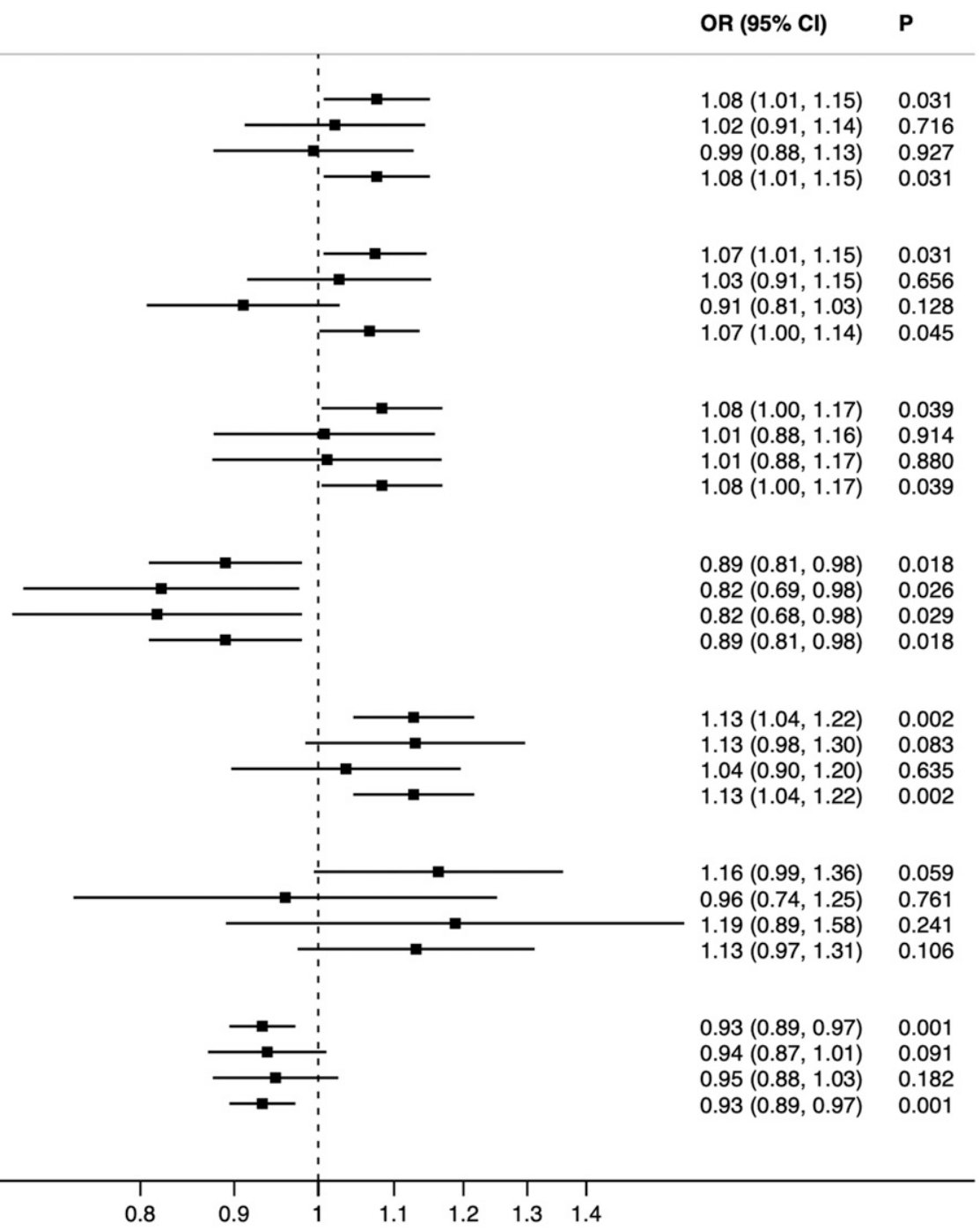

OR $(95 \% \mathrm{Cl})$ of cancer per one unit increase in log odds of T2DM

Figure 3-Sensitivity analyses of the associations between T2DM and certain site-specific cancers in UK Biobank. Heterogeneity was observed in the analysis of uterine, liver, and melanoma cancer. There was detected pleiotropy in MR-Egger analysis of cervical cancer. One and three outliers was detected and corrected in the MR-PRESSO analysis of cervical and liver cancer, respectively. IVW, inverse variance weighted.

both new-onset and long-standing T2DM facilitate the development of pancreatic cancer $(11,12)$. Pathophysiologically, this may relate to carcinogenic or cancer-promoting effects of glucose and glycation end products in reactive oxygen species generation, DNA damage, and cell proliferation $(15,43,44)$. It could also be due to the role of diabetes in the metabolic syndrome, which is associated with increased risk of pancreatic cancer $(15,45)$, or due to increased insulin levels (15). Prediabetes is characterized by a long-standing increase in insulin secretion by the $\beta$-cells of the pancreas to compensate for insulin resistance that occurs in the early stages of diabetes development. Such an increase in insulin in the pancreatic portal circulation could be carcinogenic or cancer promoting, as insulin has proliferative effects (15). It is therefore notable that we also report a positive association between FI levels and pancreatic cancer. Our findings suggest that the insulin resistance of early diabetes, in combination with hyperglycemia, may increase risk of pancreatic cancer, and importantly, this could be targeted with insulin-sensitizing agents such as metformin, which reduce such risk (46). Nevertheless, a recent MR study did not observe a positive association between diabetes and pancreatic cancer among Japanese adults. This null finding might be caused by inadequate power, since the study only had 129 pancreatic cancer cases (42).

A bidirectional relationship between T2DM and pancreatic cancer has been found in recent years $(11,15)$. Pancreatic cancer can increase diabetes risk through enhanced insulin secretion with consequent insulin resistance 


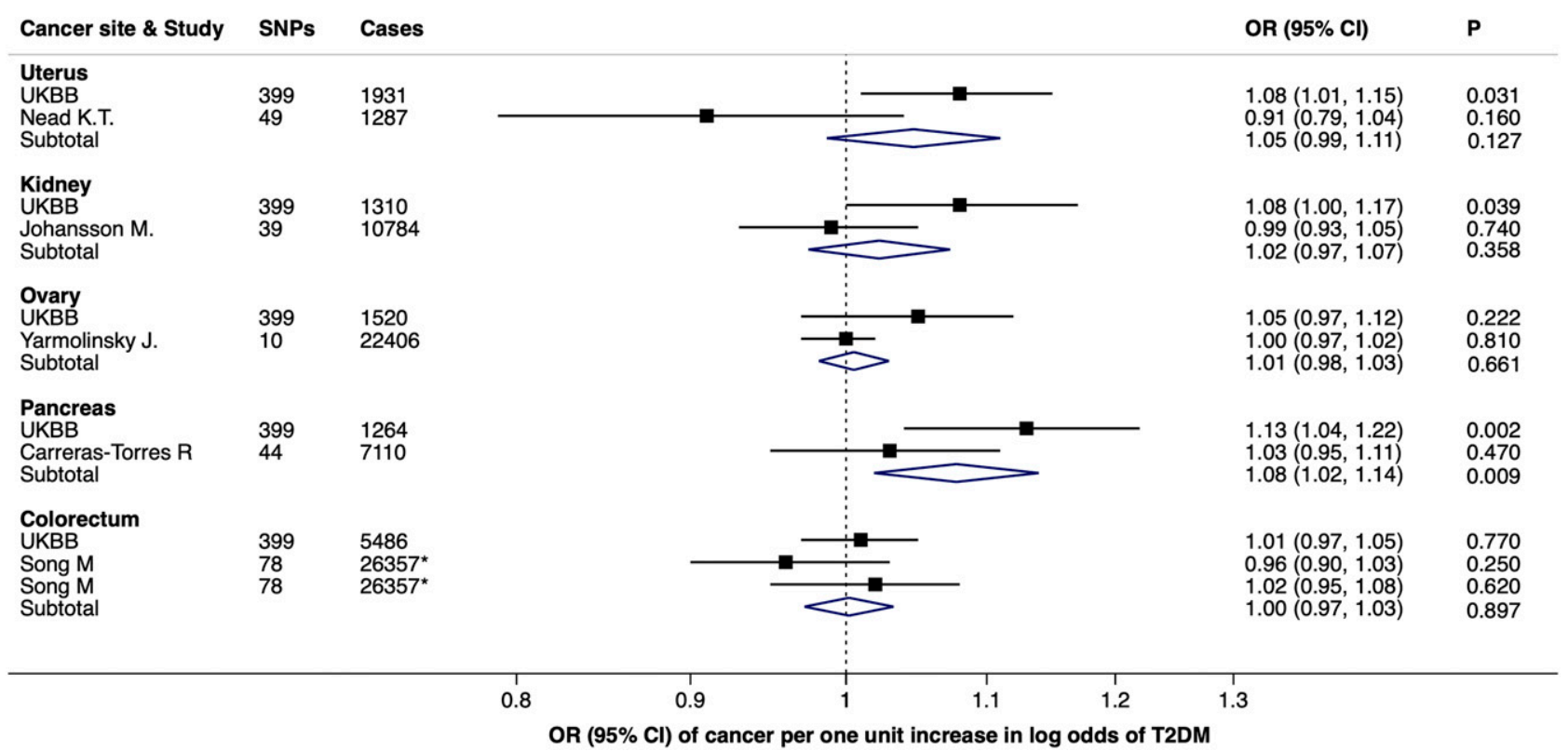

Figure 4-Meta-analysis of the association of T2DM with certain site-specific cancers. *Effect size in a study by Song et al. (34) was estimated in men and women separately. First author names appear for Cancer site \& Study column. UKBB, UK Biobank.

or due to destruction of pancreatic tissue with loss of insulin-producing $\beta$-cells. Even though several pathological features, such as insulin levels and glucose-dependent insulinotropic polypeptide levels, were different between new-onset T2DM and pancreatic cancer-caused T2DM, inaccurate classification of diabetes was common in clinical practice (15). Thus, the established observational association between T2DM and pancreatic cancer could be the result of reverse causality. The current study using MR design confirmed a causal pathway from T2DM to pancreatic cancer but also found suggestive evidence of an inverse causal pathway from pancreatic cancer to T2DM risk. This could be important clinically, and with further research the development of diabetes or prediabetes could be useful in monitoring cancer progression.

The current study also detected possible positive associations of liability to T2DM with some other site-specific cancers, with the strongest evidence for kidney, uterine, and cervical cancer. A systematic review including nine cohort studies stated that patients with diabetes had a significant increased risk of kidney cancer after adjustment for BMI and cigarette smoking (8). It could relate to increased exposure to carcinogenic or cancer-promoting growth factors or insulin-like products due to reduced excretion or be a consequence of urinary tract infections, which are common due to the relative immunosuppression seen in diabetes $(47,48)$. Similarly, the association of T2DM with uterine cancer is supported by the findings of a metaanalysis of 16 observational studies with multivariate adjustment (49). However, studies concerning the association of T2DM with cervical cancer are limited and conflicting. A retrospective cohort study with 328,994 patients with diabetes and 327,572 participants without diabetes found that newly diagnosed T2DM patients (within 3 months) had significantly increased risk of cervical cancer. However, the risk was not higher among T2DM patients after the initial 3-month period compared with risk in those without T2DM (50). In another study including 397,783 adults, the prevalence of cervical cancer was $30 \%$ higher in the group with diabetes compared with counterparts without diabetes with adjustment for age, BMI, ethnicity, lifestyle, and physical activity (51). A nationwide Australian study showed that long-term T2DM was associated with the age-standardized incidence ratio of cervical cancer but not with mortality from cervical cancer (16). Further studies are warranted to verify the causal positive association between T2DM and cervical cancer.

Our finding of an inverse association between T2DM and melanoma is in line with the findings of most but not all observational studies. A nationwide hospital-based study showed that the risk of melanoma for familial T2D patients was lower among 26,641 patients (including 125,126 T2DM patients) who had a T2DM-affected family member compared with all patients in Sweden (52). Another nationwide study in Australia also found a decreased risk of melanoma among 953,382 T2DM case subjects compared with the general Australian population (16). Nonetheless, a study with 4,501,578 veterans admitted to Veterans Affairs hospitals reported that men with diabetes had a higher risk of melanoma (53). With regard to esophageal cancer, previous findings were inconsistent. Three meta-analyses documented a positive association between T2DM and esophageal cancer; however, the results might be less robust due to substantial heterogeneity and 
potential confounding factors within the included studies $(3,54,55)$. A large-scale cohort study of $4,501,578$ black and white U.S. veterans found that male T2DM patients had a decreased risk of esophageal cancer (53). Findings of two studies focusing on T2DM and risk of esophageal cancer in Australian and Asian populations showed no association between diabetes diagnosis and risk of esophageal cancer $(16,56)$. Thus, the role of T2DM in esophageal cancer development and mortality needs more investigation.

Even though most observational studies observed a strong inverse association between T2DM and risk of prostate cancer (17), the current study provides limited evidence supporting such a causal association, which is supported by a previous MR study (25). The possible explanation for the discrepancy is anticancer effect of several drugs used for the management of T2DM, such as metformin and thiazolidinediones (57), in previous observational studies. There was suggestive evidence of a positive association of T2DM with liver cancer in the present MR study, confirming previous observational findings (3), which is likely to occur through driving nonalcoholic fatty liver disease, which can progress to hepatocellular carcinoma.

The detrimental effects of T2DM in relation to certain site-specific cancers may be driven by high insulin levels in response to insulin resistance that occurs in the development of prediabetes. It is therefore notable that we found positive associations of both T2DM and FI with pancreatic, kidney, and uterine cancer, which suggests a possible pathophysiologic mechanism. Meta-analysis of FI was also positively associated with lung cancer risk. Observational studies have proposed that hyperinsulinemia increases the risk of several cancers, such as pancreatic (58), uterine (59), gastric (60), and kidney (61) cancer, but not lung cancer (62), and insulin has multiple potential carcinogenic or cancer-promoting effects (63). Although limited evidence of an association between FG and cancer was found in the current study, except for a possible positive association with biliary tract cancer (64), hyperglycemia might play a role in the onset of certain cancers, especially liver (65) and bladder $(64,65)$ cancer. Inflammation (66), elevated hemoglobin $A_{1 c}$ levels (67), and drugs used for the management of T2DM (68) may also mediate the pathway from T2DM to cancer. Detailed mechanisms need further investigations. Further validating our findings or hypothesis, several T2DM medications have been revealed to lower the risk of common cancers, such as lung, colorectal, and breast cancer, in preclinical or clinical settings among patients with diabetes $(69,70)$. Review articles suggested that even though metformin and thiazolidinedione appeared to inhibit the proliferation and growth of certain cancer types in preclinical data, a vast majority of clinical trials have been conducted to assess the usefulness of these medications in cancer prevention and treatment $(69,70)$. Those results will facilitate the assessment of the place of metformin in cancer prevention and therapy and define the target populations.
A major strength of this study is the MR study design, which diminishes confounding and reverses causality potentially biasing the results in observational studies. In addition, we comprehensively assessed the causal associations of T2DM and related traits with overall cancer and 22 site-specific cancers using summary-level data from large genetic consortia. We conducted our study merely among European populations. Thus, the results were less likely to be biased by population stratification, but this confined the transferability of our findings to other populations. A major limitation is that the number of cases was few for several site-specific cancers, causing low precision of the estimates. Thus, it is likely that we have missed weak associations. However, we have performed a systematic review and meta-analysis to combine the data from the previous and present MR studies, thereby expanding the sample size and increasing the accuracy of the estimation as possible. Furthermore, we interpreted results relying on the consistency across three sensitivity analyses and the strengths of the associations but not the significance level (41). Even though there was heterogeneity among instrumental variables in a few analyses, no pleiotropy in the MR-Egger suggested balanced pleiotropy, which is less likely to bias the results (36). We still cannot exclude that there is any direct causal pathway from the T2DM-predisposing genetic variants to cancer. A further limitation is that we examined the liability to T2DM rather than the disease itself. Our results are therefore not fully comparable with those of observational studies where study participants have or do not have a T2DM diagnosis. Even though most of the included studies defined cancer cases based on a reliable source, such as registry and hospital/clinic data, a possible detection bias in T2DM patients may overestimate the association between T2DM and cancer. Nonetheless, considering that we examined the association of T2DM with $>20$ site-specific cancers, it is less likely that an increased or decreased chance of being diagnosed with a site-specific cancer is caused by the diagnosis of diabetes assuming no causal association between them.

\section{Conclusion}

This MR study strengthened the evidence in favor of causal associations of T2DM with increased risks of pancreatic, kidney, uterine, and cervical cancer and decreased risks of esophageal cancer and melanoma. Additionally, there was evidence of a positive association of FI levels with some overlapping cancers, which suggests that insulin resistance in early diabetes may contribute to this risk. This study lent limited support to causal associations of T2DM, FG, and FI with overall cancer risk. We suggest a higher index of suspicion for cancer and reinforcement of cancer screening recommendations among patients with T2DM to enable the early detection of cancer in this group of patients. 
Acknowledgments. Summary-level data for SNPs associated with T2DMrelated traits were extracted from DIAGRAM consortium and MAGIC. Summary-level data for genetic associations with the cancers were contributed by the BCAC, PanScan and PanC4, and UK Biobank. The analyses of UK Biobank data were conducted under application 29202. The authors thank all investigators for sharing these data.

Funding. Funding for this study came from the Swedish Research Council (Vetenskapsrådet) (grant 2019-00977). S.B. is supported by a Sir Henry Dale Fellowship jointly funded by the Wellcome Trust and the Royal Society (grant 204623/Z/16/Z). S.K. is supported by a Cancer Research UK programme grant, the Integrative Cancer Epidemiology Programme (C18281/A19169), and a Junior Research Fellowship from Homerton College, Cambridge, U.K.

Duality of Interest. No potential conflicts of interest relevant to this article were reported.

Author Contributions. S.Y. analyzed and interpreted data and wrote and reviewed the manuscript. S.K., M.V., and P.C. reviewed the manuscript. A.M.M. and S.B. prepared the data and reviewed the manuscript. S.C.L. designed the research, analyzed and interpreted the data, and reviewed the manuscript. All authors read and approved the final manuscript. S.C.L. has confirmed that the manuscript is an honest, accurate, and transparent account of the study being reported and that no important aspects of the study have been omitted. S.C.L. is the guarantor of this work and, as such, had full access to all the data in the study and takes responsibility for the integrity of the data and the accuracy of the data analysis.

\section{References}

1. Zheng Y, Ley SH, Hu FB. Global aetiology and epidemiology of type 2 diabetes mellitus and its complications. Nat Rev Endocrinol 2018;14:88-98

2. Fitzmaurice C, Allen C, Barber RM, et al.; Global Burden of Disease Cancer Collaboration. Global, regional, and national cancer incidence, mortality, years of life lost, years lived with disability, and disability-adjusted life-years for 32 cancer groups, 1990 to 2015: a systematic analysis for the Global Burden of Disease Study. JAMA Oncol 2017;3:524-548

3. Tsilidis KK, Kasimis JC, Lopez DS, Ntzani EE, loannidis JP. Type 2 diabetes and cancer: umbrella review of meta-analyses of observational studies. BMJ 2015;350:g7607

4. Guraya SY. Association of type 2 diabetes mellitus and the risk of colorectal cancer: a meta-analysis and systematic review. World J Gastroenterol 2015;21:6026-6031

5. Larsson SC, Orsini N, Wolk A. Diabetes mellitus and risk of colorectal cancer: a meta-analysis. J Natl Cancer Inst 2005;97:1679-1687

6. Wang Y, Wang B, Yan S, et al. Type 2 diabetes and gender differences in liver cancer by considering different confounding factors: a meta-analysis of cohort studies. Ann Epidemiol 2016;26:764-772

7. Graff RE, Sanchez A, Tobias DK, et al. Type 2 diabetes in relation to the risk of renal cell carcinoma among men and women in two large prospective cohort studies. Diabetes Care 2018;41:1432-1437

8. Larsson SC, Wolk A. Diabetes mellitus and incidence of kidney cancer: a meta-analysis of cohort studies. Diabetologia 2011;54:1013-1018

9. Saed L, Varse F, Baradaran HR, et al. The effect of diabetes on the risk of endometrial cancer: an updated a systematic review and meta-analysis. BMC Cancer 2019;19:527

10. Larsson SC, Mantzoros CS, Wolk A. Diabetes mellitus and risk of breast cancer: a meta-analysis. Int J Cancer 2007;121:856-862

11. Batabyal P, Vander Hoorn S, Christophi C, Nikfarjam M. Association of diabetes mellitus and pancreatic adenocarcinoma: a meta-analysis of 88 studies. Ann Surg Oncol 2014;21:2453-2462

12. Lu Y, García Rodríguez LA, Malgerud L, et al. New-onset type 2 diabetes, elevated $\mathrm{HbA} 1 \mathrm{c}$, anti-diabetic medications, and risk of pancreatic cancer. $\mathrm{Br} J$ Cancer 2015;113:1607-1614

13. Bosetti C, Rosato V, Li D, et al. Diabetes, antidiabetic medications, and pancreatic cancer risk: an analysis from the International Pancreatic Cancer CaseControl Consortium. Ann Oncol 2014;25:2065-2072

14. Ben Q, Xu M, Ning X, et al. Diabetes mellitus and risk of pancreatic cancer: A meta-analysis of cohort studies. Eur J Cancer 2011;47:1928-1937
15. Andersen DK, Korc M, Petersen GM, et al. Diabetes, pancreatogenic diabetes, and pancreatic cancer. Diabetes 2017;66:1103-1110

16. Harding JL, Shaw JE, Peeters A, Cartensen B, Magliano DJ. Cancer risk among people with type 1 and type 2 diabetes: disentangling true associations, detection bias, and reverse causation. Diabetes Care 2015;38:264-270

17. Bansal D, Bhansali A, Kapil G, Undela K, Tiwari P. Type 2 diabetes and risk of prostate cancer: a meta-analysis of observational studies. Prostate Cancer Prostatic Dis $2013 ; 16: 151-158$

18. Smith GD, Ebrahim S. 'Mendelian randomization': can genetic epidemiology contribute to understanding environmental determinants of disease? Int J Epidemiol 2003;32:1-22

19. Mahajan A, Taliun D, Thurner M, et al. Fine-mapping type 2 diabetes loci to single-variant resolution using high-density imputation and islet-specific epigenome maps. Nat Genet 2018;50:1505-1513

20. Scott RA, Lagou V, Welch RP, et al.; DIAbetes Genetics Replication and Metaanalysis (DIAGRAM) Consortium. Large-scale association analyses identify new loci influencing glycemic traits and provide insight into the underlying biological pathways. Nat Genet 2012;44:991-1005

21. Klein AP, Wolpin BM, Risch HA, et al. Genome-wide meta-analysis identifies five new susceptibility loci for pancreatic cancer. Nat Commun 2018;9:556

22. Michailidou $\mathrm{K}$, Lindström $\mathrm{S}$, Dennis $\mathrm{J}$, et al.; NBCS Collaborators; $\mathrm{ABCTB}$ Investigators; ConFab/AOCS Investigators. Association analysis identifies 65 new breast cancer risk loci. Nature 2017;551:92-94

23. Sudlow C, Gallacher J, Allen N, et al. UK Biobank: an open access resource for identifying the causes of a wide range of complex diseases of middle and old age. PLoS Med 2015;12:e1001779

24. Georgakis MK, Harshfield E, Malik R, et al. Diabetes mellitus, glycemic traits, and cerebrovascular disease: a Mendelian randomization study. 30 December 2019 [preprint]. medRxiv:2019.12.27.19015834

25. Au Yeung SL, Schooling CM. Impact of glycemic traits, type 2 diabetes and metformin use on breast and prostate cancer risk: a Mendelian randomization study. BMJ Open Diabetes Res Care 2019;7:e000872

26. Larsson SC, Scott RA, Traylor M, et al.; METASTROKE Collaboration and NINDS Stroke Genetics Network (SiGN). Type 2 diabetes, glucose, insulin, BMI, and ischemic stroke subtypes: Mendelian randomization study. Neurology 2017;89: 454-460

27. Frayling TM, Timpson NJ, Weedon MN, et al. A common variant in the FTO gene is associated with body mass index and predisposes to childhood and adult obesity. Science 2007;316:889-894

28. Nead KT, Sharp SJ, Thompson DJ, et al.; Australian National Endometrial Cancer Study Group (ANECS). Evidence of a causal association between insulinemia and endometrial cancer: a Mendelian randomization analysis. J Natl Cancer Inst 2015;107:djv178

29. Johansson M, Carreras-Torres R, Scelo G, et al. The influence of obesityrelated factors in the etiology of renal cell carcinoma-a mendelian randomization study. PLoS Med 2019;16:e1002724

30. Yarmolinsky J, Relton CL, Lophatananon A, et al. Appraising the role of previously reported risk factors in epithelial ovarian cancer risk: a Mendelian randomization analysis. PLoS Med 2019;16:e1002893

31. Carreras-Torres R, Johansson M, Gaborieau V, et al. The role of obesity, type 2 diabetes, and metabolic factors in pancreatic cancer: a Mendelian randomization study. J Natl Cancer Inst 2017;109:djx012

32. Carreras-Torres R, Johansson M, Scelo G, et al. Identifying causal risk factors of metabolic syndrome for renal cell carcinoma: a Mendelian randomization approach (Abstract). Cancer Res 2016;76(Suppl.):A4349

33. Carreras-Torres R, Johansson M, Haycock PC, et al. Obesity, metabolic factors and risk of different histological types of lung cancer: a Mendelian randomization study. PLoS One 2017;12:e0177875

34. Song M, Lu Y, Gunter M, et al. Type 2 diabetes and glycemic traits in relation to colorectal cancer risk: a Mendelian randomization study (Abstract). Cancer Res 2018;78(Suppl.):A235 
35. Bowden J, Davey Smith G, Haycock PC, Burgess S. Consistent estimation in Mendelian randomization with some invalid instruments using a weighted median estimator. Genet Epidemiol 2016;40:304-314

36. Burgess S, Thompson SG. Interpreting findings from Mendelian randomization using the MR-Egger method. Eur J Epidemiol 2017;32:377-389

37. Verbanck M, Chen CY, Neale B, Do R. Detection of widespread horizontal pleiotropy in causal relationships inferred from Mendelian randomization between complex traits and diseases. Nat Genet 2018;50:693-698

38. Forouhi NG, Luan J, Hennings S, Wareham NJ. Incidence of type 2 diabetes in England and its association with baseline impaired fasting glucose: the Ely study 1990-2000. Diabet Med 2007;24:200-207

39. Rolfe EdeL, Loos RJ, Druet C, et al. Association between birth weight and visceral fat in adults. Am J Clin Nutr 2010;92:347-352

40. Brion MJ, Shakhbazov K, Visscher PM. Calculating statistical power in Mendelian randomization studies. Int J Epidemiol 2013;42:1497-1501

41. Sterne JA, Davey Smith G. Sifting the evidence-what's wrong with significance tests? BMJ 2001;322:226-231

42. Goto A, Yamaji T, Sawada N, et al. Diabetes and cancer risk: a Mendelian randomization study. Int J Cancer 2020;146:712-719

43. Gatenby RA, Gillies RJ. Why do cancers have high aerobic glycolysis? Nat Rev Cancer 2004;4:891-899

44. Lorenzi M, Montisano DF, Toledo S, Barrieux A. High glucose induces DNA damage in cultured human endothelial cells. J Clin Invest 1986;77:322-325

45. Rosato V, Tavani A, Bosetti C, et al. Metabolic syndrome and pancreatic cancer risk: a case-control study in Italy and meta-analysis. Metabolism 2011;60: 1372-1378

46. Wang Z, Lai ST, Xie L, et al. Metformin is associated with reduced risk of pancreatic cancer in patients with type 2 diabetes mellitus: a systematic review and meta-analysis. Diabetes Res Clin Pract 2014;106:19-26

47. Lindblad $\mathrm{P}$, Chow $\mathrm{WH}$, Chan $\mathrm{J}$, et al. The role of diabetes mellitus in the aetiology of renal cell cancer. Diabetologia 1999;42:107-112

48. Werner $\mathrm{H}$. Tumor suppressors govern insulin-like growth factor signaling pathways: implications in metabolism and cancer. Oncogene 2012;31:2703-2714 49. Friberg E, Orsini N, Mantzoros CS, Wolk A. Diabetes mellitus and risk of endometrial cancer: a meta-analysis. Diabetologia 2007;50:1365-1374

50. Johnson JA, Bowker SL, Richardson K, Marra CA. Time-varying incidence of cancer after the onset of type 2 diabetes: evidence of potential detection bias. Diabetologia 2011;54:2263-2271

51. Li C, Balluz LS, Ford ES, Okoro CA, Tsai J, Zhao G. Association between diagnosed diabetes and self-reported cancer among U.S. adults: findings from the 2009 Behavioral Risk Factor Surveillance System. Diabetes Care 2011;34:13651368

52. Hemminki K, Li X, Sundquist J, Sundquist K. Risk of cancer following hospitalization for type 2 diabetes. Oncologist 2010;15:548-555

53. Atchison EA, Gridley G, Carreon JD, Leitzmann MF, McGlynn KA. Risk of cancer in a large cohort of U.S. veterans with diabetes. Int J Cancer 2011;128:635-643
54. Xu B, Zhou X, Li X, Liu C, Yang C. Diabetes mellitus carries a risk of esophageal cancer: a meta-analysis. Medicine (Baltimore) 2017;96:e7944

55. Huang W, Ren H, Ben Q, Cai Q, Zhu W, Li Z. Risk of esophageal cancer in diabetes mellitus: a meta-analysis of observational studies. Cancer Causes Control 2012;23:263-272

56. Cheng KC, Chen YL, Lai SW, Tsai PY, Sung FC. Risk of esophagus cancer in diabetes mellitus: a population-based case-control study in Taiwan. BMC Gastroenterol 2012;12:177

57. De Nunzio C, Tubaro A. Prostate cancer: diabetes and prostate cancer-an open debate. Nat Rev Urol 2013;10:12-14

58. Stolzenberg-Solomon RZ, Graubard BI, Chari S, et al. Insulin, glucose, insulin resistance, and pancreatic cancer in male smokers. JAMA 2005;294:2872-2878 59. Gunter MJ, Hoover DR, Yu H, et al. A prospective evaluation of insulin and insulin-like growth factor-I as risk factors for endometrial cancer. Cancer Epidemiol Biomarkers Prev 2008;17:921-929

60. Hidaka A, Sasazuki S, Goto A, et al.; JPHC Study Group. Plasma insulin, C-peptide and blood glucose and the risk of gastric cancer: the Japan Public Health Center-based prospective study. Int J Cancer 2015;136:1402-1410

61. Spyridopoulos TN, Dessypris N, Antoniadis AG, et al.; Obesity and Cancer Oncology Group. Insulin resistance and risk of renal cell cancer: a case-control study. Hormones (Athens) 2012;11:308-315

62. Balkau B, Kahn HS, Courbon D, Eschwège E, Ducimetière P; Paris Prospective Study. Hyperinsulinemia predicts fatal liver cancer but is inversely associated with fatal cancer at some other sites: the Paris Prospective Study. Diabetes Care 2001;24:843-849

63. Godsland IF. Insulin resistance and hyperinsulinaemia in the development and progression of cancer. Clin Sci (Lond) 2009;118:315-332

64. Larsson SC, Giovannucci EL, Wolk A. Sweetened beverage consumption and risk of biliary tract and gallbladder cancer in a prospective study. J Natl Cancer Inst 2016;108:djw125

65. Rapp K, Schroeder J, Klenk J, et al. Fasting blood glucose and cancer risk in a cohort of more than 140,000 adults in Austria. Diabetologia 2006;49:945-952 66. Grossmann V, Schmitt VH, Zeller T, et al. Profile of the immune and inflammatory response in individuals with prediabetes and type 2 diabetes. Diabetes Care 2015;38:1356-1364

67. Goto A, Noda M, Sawada N, et al.; JPHC Study Group. High hemoglobin A1C levels within the non-diabetic range are associated with the risk of all cancers. Int $J$ Cancer 2016;138:1741-1753

68. Coyle C, Cafferty FH, Vale C, Langley RE. Metformin as an adjuvant treatment for cancer: a systematic review and meta-analysis. Ann Oncol 2016;27:21842195

69. Colmers IN, Bowker SL, Johnson JA. Thiazolidinedione use and cancer incidence in type 2 diabetes: a systematic review and meta-analysis. Diabetes Metab 2012;38:475-484

70. Kasznicki J, Sliwinska A, Drzewoski J. Metformin in cancer prevention and therapy. Ann Transl Med 2014;2:57 